\title{
Diversidad y conservación de Catasetum (Orchidaceae: Catasetinae) en el Departamento de Santander, Colombia
}

\author{
Diversity and Conservation of Catasetum (Orchidaceae: Catasetinae) in \\ Department of Santander, Colombia
}

\author{
Miguel Macgayver Bonilla Morales ${ }^{\mathrm{a}}$ \\ Adriana Carolina Aguirre Morales ${ }^{\mathrm{b}}$ \\ Holguer López Toscano ${ }^{c^{*}}$
}

Recepción: 28-oct-2015

Aceptación: 28-abr-2016

\begin{abstract}
Resumen
Catasetum tiene distribución Neotropical y su centro de diversidad está en la Amazonía brasileña. Colombia, pese a ser uno de los países más ricos en orquideoflora, es uno de los menos explorados. Por tal razón, el objetivo de la investigación fue conocer la diversidad, distribución, conservación y especies con potencial de uso en cultivo comercial de Catasetum para el departamento de Santander y Colombia en general. Se identificaron 14 especies y un nuevo registro para Colombia, C. tenebrosum. Tales taxones han experimentado un proceso de cultivo y domesticación para la preservación y propagación del material, con el propósito de manejo en colecciones privadas y comercialización. La distribución y riqueza interespecífica de Catasetum se encuentra principalmente en los municipios asociados al Valle interandino del Magdalena medio, adicional a esto, la modelación de nicho ecológico, incluye un área pequeña hacia el norte de la cordillera Oriental, flanco occidental, en la región del piedemonte como un foco donde se concentra las mejores condiciones bioclimáticas, entre un rango altitudinal de 50 a $1000 \mathrm{~m}$. Se amplía la distribución y rango altitudinal de $C$. lucis, especie exclusiva de Santander, y en este estudio no se encontraron poblaciones nativas de C. tricorne. En conclusión, el departamento de Santander tiene la mayor diversidad de Catasetum en Colombia, por lo que puede ser considerado centro de conservación in situ y ex situ de las especies del país.
\end{abstract}

Palabras clave: Catasetum, Orchidaceae, Modelación de nicho ecológico, Valle interandino.

\footnotetext{
Abstract

Catasetum has neotropical distribution and its diversity center is in the Brazilian Amazon. Spite of, Colombia being one of the richest countries in orchid flora, also it has been one of the least explored. For this reazon, the objective of this research was to understand the diversity, distribution, conservation and species with potential use in commercial cultivation of Catasetum to Santander Department and Colombia in general. 14 species and a new report for Colombia, C. tenebrosum were recorded. These plants have undergone a

${ }^{a}$ Lic. Pdn. Agrop., M.Sc Biológicas, Facultad de Ciencias Agropecuarias, Universidad Nacional de Colombia-Sede Palmira.

${ }^{b}$ I.A, cM.Sc Biológicas, Facultad de Ciencias Agropecuarias, Universidad Nacional de Colombia-Sede Palmira.

${ }^{\mathrm{c}}$ Fotógrafo profesional de la Biodiversidad, Asorquisan de Bucaramanga-Santander.

*Autor de correspondencia: mmbonillam@unal.edu.co
} 
process of cultivation and domestication, for the material preservation and propagation, with the aim to manage private collections and marketing.

The Catasetum distribution and inter-species wealth is mainly related with municipalities of the interandino Middle Magdalena Valley, stands like ecological niche modeling, adding the Eastern Cordillera, Occidental flank in the region of Piedmont, between an altitude ranges of 50 to $1000 \mathrm{~m}$. It marks the distribution and altitudinal range $C$. lucis, Santander exclusive specie. In this study the $C$. tricorne native populations were not found. In conclusion, the Santander Department has the greatest diversity of Catasetum in Colombia, so it can be considered a species conservation center in situ and ex situ in the country.

Key words: Catasetum, Orchidaceae, Ecological Niche Modeling, Inter-Andean Valley.

\section{Introducción}

Orchidaceae Juss. es considerado uno de los grupos más diversos, con ca. 28,000 especies y 736 géneros [1,2]. Colombia cuenta con cerca de 4,270 especies de orquídeas distribuidas en 274 géneros, donde la mayor parte contienen muy pocas especies, ya que el $71 \%$ de los géneros presentan menos de 10 spp. [3]. En algunos casos, las especies endémicas que son 1,572, tan sólo se conocen del espécimen tipo en colecciones botánicas, y en otros, no registran el sitio de colecta ya que provinienen de cultivo, por lo que trabajos realizado en el siglo XXI han permitido conocer la distribución y otros aspectos ecológicos de algunas de ellas, e.g., Catasetum rectangulare [3, 4].

Colombia cuenta con seis géneros de los siete que posee la subtribu Catasetinae Lindl.: Catasetum Rich. ex Kunth, Clowesia Lindl., Cycnoches Lindl., Dressleria Dodson, Galeandra Lindl. y Mormodes Lindl.; de estos, el primero es el más diverso y abundante, y tiene la mayor distribución en el país, particularmente en la región Andina, la Amazonía y la Orinoquía [5, 6]. Las especies de Catasetum pueden ser epifitas, saprófitas, terrestres o litófitas, están asociadas, principalmente, desde su germinación, crecimiento y desarrollo a interacciones micorrízicas y mimercófilas para su defensa, y en cuanto a su polinización, a las abejas Euglossini $[6,7]$.

En su mayoría, las especies de Catasetum han experimentado un proceso de cultivo relacionado con el interés que causan sus flores [8], particularmente, las unisexuales masculinas o estaminadas, ya que las femeninas presentan, generalmente, homología floral interespecífica y tienen bajo interés por su forma. Adicionalmente, el uso de las flores estaminadas, por la diversidad interespecífica del grupo, y de las flores pistiladas, que presenta homología y receptividad, posibilita el flujo genético y la generación de híbridos tanto naturales como artificiales en mejora de la forma, los colores, la precocidad y la permanencia en la floración, con fines comerciales $[9,10]$.

Santander es considerado uno de los departamentos que alberga la mayor diversidad florística de orquídeas en Colombia, con aproximadamente 570 especies [11]; sin embargo, el conocimiento de estas todavía es preliminar, y muchas de sus especies están por descubrirse. De acuerdo con los estudios realizados por Ortiz y Arango [12], Ortiz [13], Ortiz y Uribe [14-15], Bernal et al. [16] se conocen de 29 a 34 especies del género Catasetum para Colombia, y cifras actuales alcanzan 38 especies en el país [4]. El trabajo de López [17] en Orquídeas Nativas de Santander registra 14 especies del género Catasetum; sin embargo, este primer e importante compendio de orquideoflora para el departamento registra fotos y nombres de las especies, con diferentes confusiones. Por tal motivo, la presente investigación tuvo dentro de sus objetivos determinar los taxones de Catasetum, aspectos de manejo y cultivo asociados a la comercialización, su distribución y rangos altitudinales preferentes y la diversidad y la riqueza intra/interespecífica, que permita conocer aspectos ecogeográficos básicos del grupo, con la finalidad de establecer a futuro programas de manejo, conservación y cultivo en Santander como especies relacionadas y de ocupación en Colombia.

\section{Metodología}

\section{1. Área de estudio}

El estudio se realizó en el departamento de Santander, Colombia, nororiente de los Andes colombianos. El departamento está conformado por 87 municipios, entre 250 y $4000 \mathrm{msnm}$, centrada la ubicación en el punto $05^{\circ} 42^{\prime} 34$ " y $08^{\circ} 07^{\prime} 32^{\prime \prime}$ de longitud oeste, con una temperatura que oscila entre $6{ }^{\circ} \mathrm{C}$ y $30{ }^{\circ} \mathrm{C}$. 
Tabla 1. Lista de especies de Catasetum para Santander, Colombia. * Endémica de Santander, **Endémica de Colombia.

\begin{tabular}{|c|c|c|c|c|}
\hline Especie & $\begin{array}{c}\text { Altitud } \\
\text { (m.s.n.m.) }\end{array}$ & Endémica & $\begin{array}{l}\text { Silvestre (S)/ } \\
\text { Cultivada (C) }\end{array}$ & $\begin{array}{c}\text { Epifitas (E)/ } \\
\text { Terrestre (T) }\end{array}$ \\
\hline Catasetum aff. barbatum (Lindl.) Lindl. & $50-700(1200)$ & No & $\mathrm{S} / \mathrm{C}$ & $\mathrm{T}$ \\
\hline Catasetum lucis P. Ortiz \& G. Arango & $770(1200)$ & $\mathrm{Si}^{*}$ & $\mathrm{~S} / \mathrm{C}$ & $\mathrm{E}$ \\
\hline Catasetum macrocarpum Rich. ex Kunth & $50-400(939)$ & No & $\mathrm{S} / \mathrm{C}$ & $\mathrm{E}$ \\
\hline Catasetum maculatum Rich. ex Kunth & $400-1200$ & No & $\mathrm{S} / \mathrm{C}$ & $\mathrm{E}$ \\
\hline Catasetum naso Lindl. & $800-1200$ & No & $\mathrm{S} / \mathrm{C}$ & $\mathrm{E}$ \\
\hline Catasetum planiceps Lindl. & $50-700$ & No & $\mathrm{S} / \mathrm{C}$ & $\mathrm{T}$ \\
\hline Catasetum saccatum Lindl. & $200-900$ & No & $\mathrm{S} / \mathrm{C}$ & $\mathrm{E}$ \\
\hline Catasetum sanguineum Lindl. \& Paxton & $800-1200$ & No & $\mathrm{S} / \mathrm{C}$ & $\mathrm{E} / \mathrm{T}$ \\
\hline Catasetum sp. & $800-1200$ & & $\mathrm{~S} / \mathrm{C}$ & $\mathrm{E}$ \\
\hline Catasetum tabulare Lindl. & $400-1000$ & $\mathrm{Si}^{* *}$ & $\mathrm{~S} / \mathrm{C}$ & $\mathrm{E}$ \\
\hline Catasetum tenebrosum Kraenzl. & $500-900$ & No & $\mathrm{S} / \mathrm{C}$ & $\mathrm{E}$ \\
\hline Catasetum tricorne P. Ortiz & $700-1100$ & $\mathrm{Si}^{*}$ & $\mathrm{~S} / \mathrm{C}$ & $\mathrm{E}$ \\
\hline Catasetum tuberculatum Dodson & $300-1000$ & No & $\mathrm{S} / \mathrm{C}$ & $\mathrm{E}$ \\
\hline Catasetum viridiflavum Hook. & $0-400$ & No & $\mathrm{S} / \mathrm{C}$ & $\mathrm{E}$ \\
\hline
\end{tabular}

\subsection{Herbarios y literatura}

Se visitaron y revisaron colecciones de herbarios nacionales (COL, CDMB, HPUJ, JAUM y UIS). Además, se consultaron herbarios en línea, como MO y KEW, sumado a la revisión de literatura de orquídeas de Colombia relacionada con el departamento de Santander [3, 5, 9, 11-18]. Para la identificación taxonómica de las especies se contó con la validación de protólogos, espécimen tipo y expertos en el grupo. En la corroboración de la información asociada al cultivo de las especies encontradas se tuvo de referente a Holst [10].

\subsection{Fase de campo}

Del 2008 al 2015 se realizaron salidas de campo en municipios de Santander donde se visitaron áreas boscosas y caminos; además, se accedió al conocimiento de los cultivadores de la región y de las asociaciones orquideológicas. A cada una de las especies encontradas se les tomó registro fotográfico, flor (antoteca) y datos de georreferenciación, y, en algunos casos, se recibieron donaciones de plantas por parte de los cultivadores como espécimen de herbario, con la finalidad de su posterior identificación.

\subsection{Distribución y modelación de nicho climático}

Con la información obtenida se constituyó una base de datos en Excel 2010, para análisis básicos [4]. Los datos fueron analizados con el programa DIVA-GIS@ versión 7.5, con la finalidad de establecer la distribución mediante puntos en el mapa de
Santander [19]; luego, se usó el modelo climático de ocurrencias de especies BIOCLIM que utiliza el paquete WorldClim en DIVA-GIS $\odot$ para determinar las áreas de preferencias [20], generando los mapas que se editaron con el mismo software.

\subsection{Conservación}

En cuanto a los aspectos de conservación, se tuvieron en cuenta los criterios planteados por la IUCN [21] y los asociados por Calderón [22] para establecer el estatus de conservación de orquídeas colombianas.

\section{Resultados y discusión}

\subsection{Inventario florístico}

Para el departamento de Santander se registran 14 especies de Catasetum (tabla 1); dentro de estas, un taxón que está en proceso de determinación. López [17] registra la misma cifra, pese a la existencia en su publicación de especies indebidamente determinadas o confundidas con flores femeninas y que no se incluyen dentro de este inventario, como las siguientes: $C$. collare Cogn., C. discolor (Lindl.) Lindl. y C. maroaense G.A. Romero \& C. Gómez. En recopilación de fotografías, Ortiz y Uribe [14] establecen para Colombia alrededor de 29 especies de Catasetum, sin especificar colecciones, localidad, departamentos $\mathrm{u}$ otra información que permita relacionarlas. Datos más recientes de Bonilla et al. [4, 5] registran 38 especies e incluyen aspectos preliminares de distribución y taxonomía; de tal manera, Santander 
posee, aproximadamente, el $37 \%$ de las especies de Catasetum que se encuentran en el país.

La lista de especies registradas en Santander comprende individuos hallados en zonas nativas y algunos que han experimentado un proceso de domesticación por cultivadores, coleccionistas e interesados en la ornamentación de jardines; lo que sugiere diferentes valores de uso asociados tanto al cultivo como al estudio biológico del grupo y la comercialización $[8,10]$.

En el estudio realizado para la constitución del Catálogo de Plantas y Líquenes de Colombia, el género Catasetum registra el mayor número conocido en inventario para el país; sin embargo, referente a las localidades de las especies listadas para Santander solo se presentan registros de las dos especies endémicas: C. lucis y C. tricorne [16]; por tal razón, el inventario de la tabla 1 define las especies que se encuentran en zonas naturales en Santander, como su uso actual en cultivo por su valor ornamental.

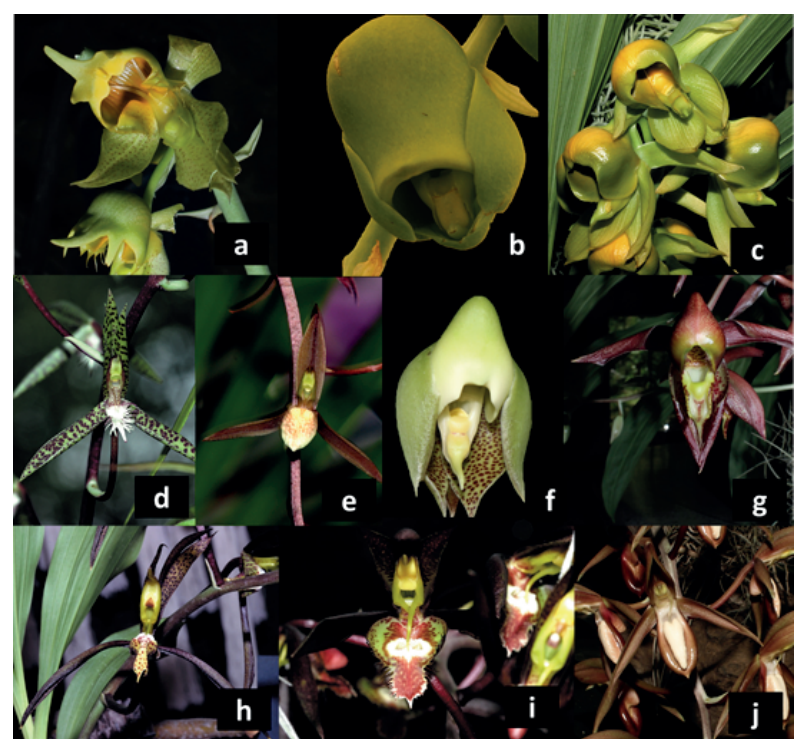

Figura 1. Catasetum de Santander: a) C. sanguineum, b) C. planiceps, c) C. viridiflavum, d) C. aff. barbatum, e) C. tuberculatum, f) C. macrocarpum, g) C. maculatum, h-i) C. saccatum, j) C. tabulare.

\subsection{Diversidad silvestre y cultivada}

Todas las especies encontradas en el presente estudio tienen un proceso de propagación y domesticación a nivel nacional o internacional, a través del cultivo por coleccionista o con fines de comercialización local (figura 1); sin embargo, C. naso, $C$. tenebrosum y $C$. viridiflavum son solamente halladas en forma silvestre en Santander, sin colecciones en cultivo, aunque C. tenebrosum y C. viridiflavum son fácilmente cultivadas en otros países; por su parte las colecciones de $C$. naso son escasas y ampliamente confundidas con C. sanguineum [10]. C. viridiflavum ha sido cultivada en viveros de Antioquia con óptimo crecimiento. Por otro lado, C. tricorne es una especie que se reportó como nueva de cultivo [13] y no se ha vuelto a conocer en colecciones o publicaciones desde la colección tipo; de tal manera que no se registran poblaciones o individuos silvestres que permitan determinar aspectos ecológicos sobre esta especie.

Otras especies, como $C$. planiceps, $C$. saccatum y $C$. tuberculatum son raramente encontradas bajo cultivo en Santander, aunque su proceso de adaptación y manejo ex situ no es complejo. C. sanguineum es una especie que se puede encontrar terrestre y epifita, sembrada en jardines con alta variabilidad morfológica asociada a cambios cuantitativos en el ápice del labelo, principalmente. Esta especie ha sido estudiada en Venezuela, donde se conocen más aspectos relacionados con su cultivo [23]. C. aff. barbatum, C. macrocarpum y C. tabulare han experimentado un proceso de domesticación más amplio en Colombia por interesados en el manejo y cultivo de la Catasetinoflora [6]. Sin embargo, en países de Sur y Centroamérica estas especies son ampliamente cultivadas y utilizadas en cruzamientos específicos para la formación de híbridos de interés comercial $[10,24]$. La especie más abundante en forma silvestre y cultivada es $C$. maculatum, ya que sus flores presentan diferentes y llamativos colores para los coleccionistas y su manejo, crecimiento y adaptación ex situ resulta sencillo.

Se incluye un nuevo reporte para Colombia: $\mathrm{Ca}$ tasetum tenebrosum Kraenzl, encontrado en Puerto Wilches, Magdalena Medio, a 500 m.s.n.m. sobre una palma (figura 2). Esta especie ha sido registrada para Ecuador y Perú entre 500 y 1800 m.s.n.m. [10], pero hasta ahora no se había encontrado una población natural en Colombia. Catasetum tenebrosum Kraenzl ha sido cultivada en diferentes países por sus flores llamativas y posibilidades de hibridación. 

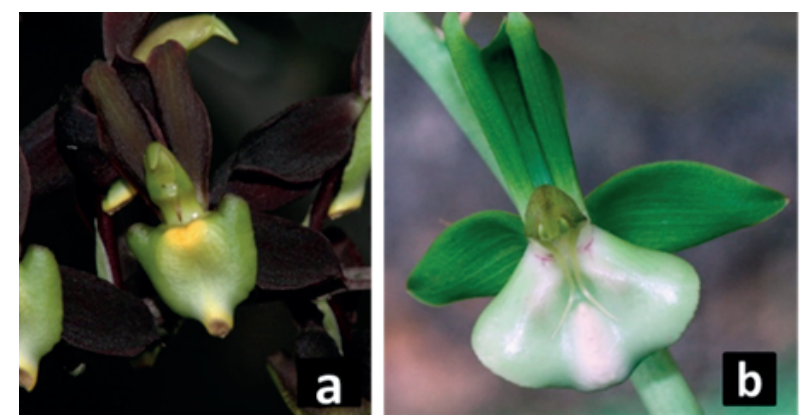

Figura 2. Especies de Catasetum en Santander. a) C. tenebrosum, un nuevo registro, y b) C. lucis, una especie endémica de Santander.

Existen dos especies endémicas para Colombia y exclusivas para el departamento de Santander: $C$. lucis [12] y C. tricorne [13]. En este estudio, C. lucis ha sido encontrada en poblaciones naturales y en cultivo (figura 2b). Ortiz y Arango [12] determinaron dos formas de esta especie: el autónimo C. lucis $\mathrm{f}$. lucis, y C. lucis f. tigrinum, este último posiblemente debe su nombre a algún tipo de alelismo múltiple relacionado con el color florarl, pues la expresión se puede presentar en diferentes individuos [25]. Sin embargo, las flores poseen caracteres morfológicos cuantitativos y cualitativos con pocas varianzas considerable en la diferenciación infraespecífica; adicionalmente, ambas formas se encuentran creciendo muy cerca por lo que comparten distribución; de tal manera, no existe una barrera geográfica que los separe.

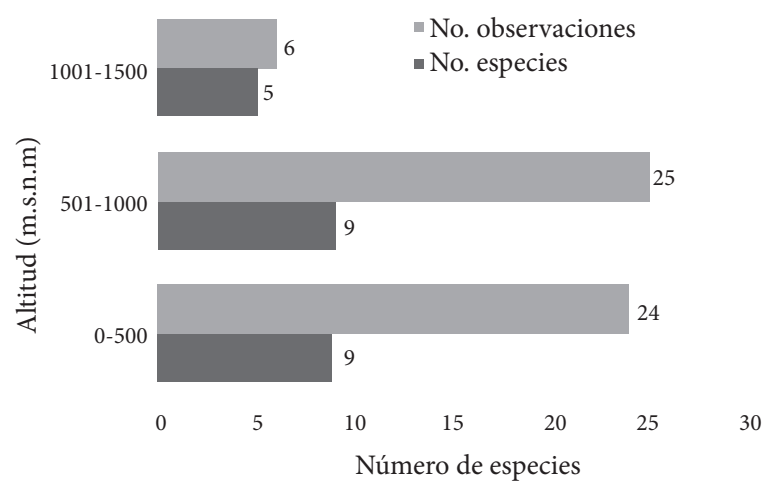

Figura 3. Diversidad altitudinal vs. número de especies de Catasetum en Santander, Colombia.

La figura 3 muestra la diversidad altitudinal de Catasetum en el departamento de Santander. Los rangos de 0 a 500 y 501 a $1,000 \mathrm{~m}$. presentan un registro de nueve especies (de 14 registradas) con un número de observaciones 49 individuos en poblaciones nativas. Sin embargo, cuando se realizan exploraciones en zonas más altas, que comprenden de 1,001 a 1,500 m, el número de especies disminuye, encontrándose dos especies nativas, exclusivas de la zona: $C$. lucis y $C$. tricorne, particularmente asociadas a la región Andina. Catasetum agrupa especies que por sus características fisiológicas, de pseudobulbo engrosado con la capacidad de almacenamiento de agua y nutrientes y hojas plisadas, se encuentran principalmente relacionadas con el clima cálido de tierras bajas $[9,18]$ y con sus polinizadores: las abejas Euglossini que presentan principalmente mayor diversidad y abundancia en estos rangos [8]. Por consiguiente, las zonas de exploración y de estudios deben comprender particularmente los rangos de 0 a 500 y 501 a 1,000 m., para el establecimiento de las especies en colecciones personales o cultivos comerciales.

\subsection{Distribución y modelación de nicho climático}

Las especies de Catasetum se distribuyen principalmente al norte del Valle interandino del río Magdalena, conocido como Magdalena medio, y en el flanco occidental de la cordillera Oriental, entre los municipios de Girón, Lebrija, Bucaramanga y Rionegro (figura 4); esto se asocia a lo determinado por López [17] en cuanto a la distribución de Catasetum en Santander. Adicionalmente, los patrones de distribución del género en el país se asocian a la ecología de los valles interandinos, piedemonte bajo andino y sabanas, donde crecen poblaciones en árboles con corteza fragmentada o caídos, en descomposición, como en palmas [26], aunque la mayor abundancia y diversidad está en el Amazonas, que es una zona todavía poco explorada. Además, se presenta un vacío de colecta (GAP), que involucra la zona más diversa del grupo, que es el costado inferior izquierdo del Magdalena medio en Santander. De tal manera, este sitio es donde probablemente existan especies desconocidas, ya que cumple las condiciones ecológicas asociadas a las registradas para otras especies en Santander. En Colombia, tanto para Catasetum como para grupos asociados a la subtribu Catasetinae, se presentan diferentes GAP en sitios estratégicos donde se predice la ocurrencia de especies desconocidas [26]. 


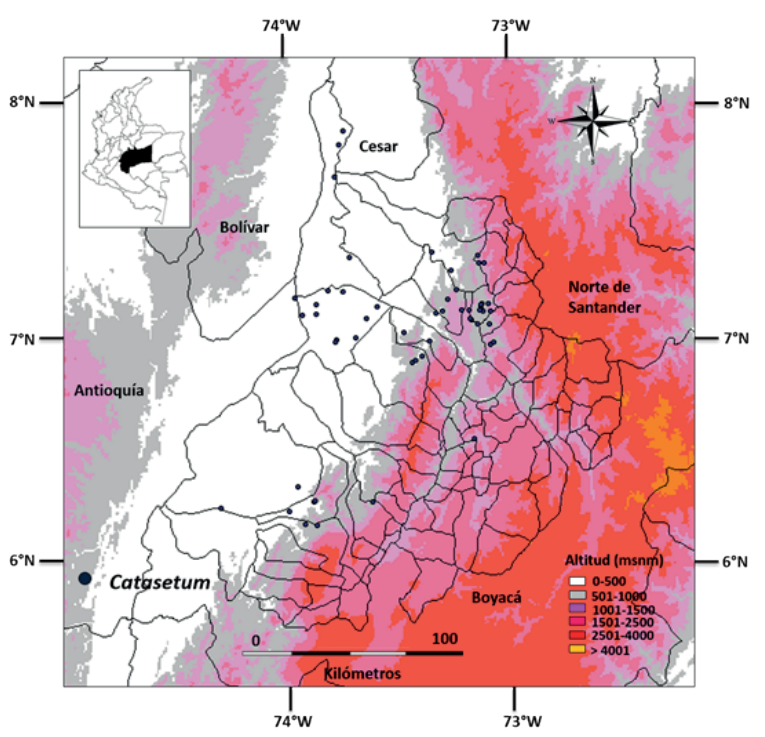

Figura 4. Distribución de Catasetum en Santander, Colombia.

El mayor número de especies y su abundancia se concentran en los puntos 1 (presencia de especies hacia el noroccidente) y 2 (presencia de especies hacia el suroccidente), definidos por su agrupación y cercanía en el mapa de distribución asociados principalmente a zonas de vida del Valle interandino y piedemonte andino. De acuerdo con análisis espaciales de la subtribu Catasetinae para Colombia, en esta región se concentran diversas poblaciones interespecíficas del grupo [26].

Catasetum aff. barbatum, C. macrocarpum, $C$. naso, C. planiceps, C. sanguineum, C. saccatum, C. tabulare, $C$. tenebrosum y $C$. tuberculatum son especies reportadas principalmente en el espacio interandino de las cordilleras Oriental y Central, la Orinoquía y la Amazonía colombiana y aledañas a Brasil, Perú y Venezuela $[9,18,26]$, motivo por el que su aparición debió suceder antes del último levantamiento andino, que hace relación a la cordillera Oriental andina colombiana. En cuanto a $C$. viridiflavum, es una especie más relacionadas con Centroamérica [27], al igual que C. maculatum. Especies restringidas en área geográfica para Santander están $C$. lucis y $C$. tricorne. De acuerdo con Ortiz y Arango [12], C. lucis se conocía solo del municipio de Girón, pero el presente estudio amplía su distribución a Bucaramanga, Floridablanca, Lebrija, Matanza y Piedecuesta. C. tricorne es conocida de la localidad tipo (Girón) y ha sido cultivaba en Bucaramanga.
La modelación de nicho ecológico de las especies de Catasetum de Santander involucra, principalmente, el Valle Interandino del Magdalenta medio y el flanco occidental de la cordillera Oriental hacia la región del Piedemonte (figura 5); zonas en las que se han realizado colectas de campo para algunas especies de Catasetum [17], por lo que las preferencias climáticas convergen con los puntos de observación del grupo. Sin embargo, la modelación de nicho ecológico potencial involucra además zonas donde no se ha registrado la presencia de especies, por lo que pueden ser espacios que permitan ampliar los conocimientos de las especies ya existentes, así como de otras que se encuentran en departamentos fronterizos que involucran principalmente Antioquía, Bolívar, Boyacá y Cesar.

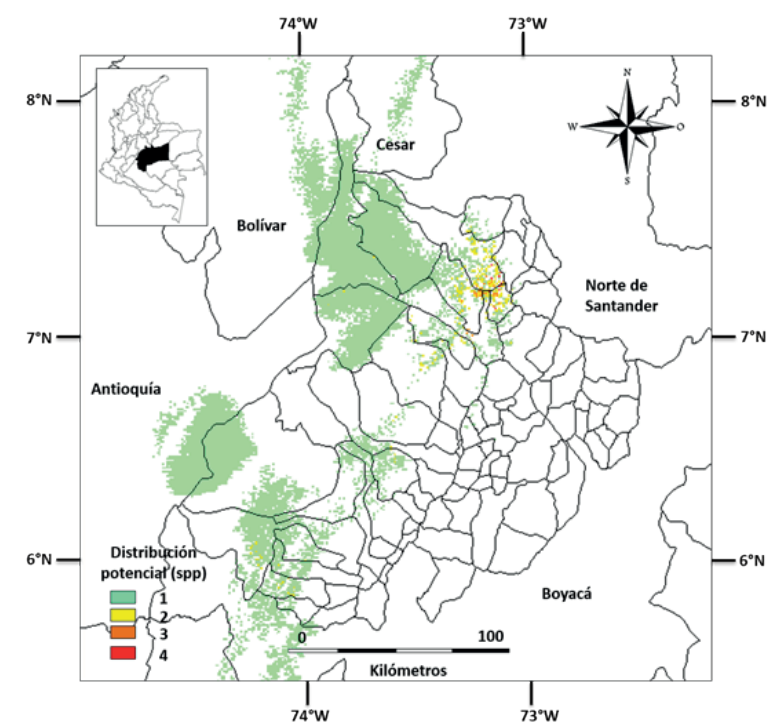

Figura 5. Modelación de nicho climático de Catasetum en Santander.

\subsection{Conservación}

Colombia no cuenta con planes de conocimiento y conservación de orquídeas, pues hasta el presente solo se conoce el estado de conservación de aproximadamente 371 especies [22], siendo menos del $10 \%$ de la orquideoflora del país [3, 28]. Las especies $C$. aff. barbatum, C. saccatum, $C$. tenebrosum y C. tuberculatum son, generalmente, de amplia distribución y en su mayoría cultivadas por coleccionistas. Sin embargo, debido a la extracción desmesurada y selectiva de individuos o poblaciones de su estado silvestre se pueden considerar en estado de Casi Amenazada (NT); por su parte, C. macrocarpum y C. maculatum son categorizadas en Preocupación 
Menor (LC), por su amplio uso y la ocurrencia en bosques nativos (figura 6).

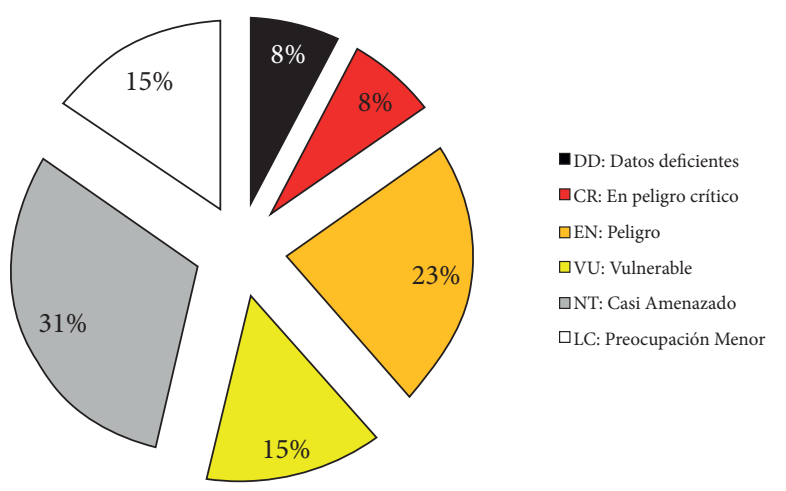

Figura 6. Estatus de conservación de las especies de Catasetum para Santander.

C. planiceps y $C$. naso se hallan en la categoría de Vulnerable (VU), por su tamaño y número de poblaciones in situ. C. sanguineum, de la frontera entre Colombia y Venezuela; $C$. viridiflavum, de $\mathrm{Pa}-$ namá, Colombia y Perú, pero con pocas poblaciones y restringidas a zonas especifica en estos dos últimos países, y C. tabulare, endémica de los Valles interandinos colombianos, son especies de distribución reducida y en áreas nativas vulnerables, por lo que se califican En Peligro (EN).

De acuerdo con los literales de los Criterios de la Lista Roja [21, 22], C. tricorne se categoriza con Datos Deficientes (DD) y C. lucis, En Peligro Crítico (CR). C. tricorne solo es conocida del espécimen tipo (Girón, Santander) de una planta cultivada, y es muy afín a $C$. triodon Rchb. f., pero esta especie ha sido reportada para Brasil [12]. C. lucis, especie exclusiva de Santander [13], se ha encontrado en diferentes localidades, pero el número de individuos silvestres no supera los 10; por tal motivo, su área de ocupación es muy restringida; además, ambas especies se encuentran en áreas desprotegidas y su población es reducida y fragmentada; el área de ocupación es $<100 \mathrm{~km}^{2}$ y las poblaciones conocidas tienen $<50$ individuos maduros. No obstante, $C$. lucis es una especie que ha experimentado cultivo y domesticación por coleccionistas. Además, las asociaciones orquideológicas del departamento, por ejemplo Asorquisan, han empezado a cultivar algunas especies que se encuentran nativas en fincas y viveros, como $C$. lucis; así, el cultivo como estrategia de conservación (conservación in situ on farm) no solo va a permitir mantener el patrimonio genético de una especie, sino su multiplicación y preservación.

\section{Conclusiones}

La exploración en municipios aledaños al Magdalena medio, asociados a los GAP establecidos, va a permitir conocer un poco más la diversidad de Catasetum de Santander. Dentro de las especies estudiadas, $C$. tricorne no se encontró en este estudio, de tal manera que futuras investigaciones deben estar enfocadas a su búsqueda, ya que es una especie endémica de Colombia.

C. lucis y C. tricorne son especies exclusivas de Santander (Colombia), por tal motivo, desarrollar planes para su conocimiento, manejo y conservación tanto in situ como ex situ on farm (asociando cultivadores) es urgente como necesario; además, el departamento de Santander, por concentrar el mayor número de especies de Catasetum en el país, es un sitio estratégico como centro de conservación in situ y ex situ de este grupo para Colombia.

\section{Agradecimientos}

A los directores y encargados de las colecciones botánicas en Orchidaceae de herbarios: Humberto Emilio García Pinzón (UIS), Alicia Rojas (CDMB), Julio Betancur (COL), Néstor García (HPUJ) y Álvaro Cogollo (JAUM), por permitir revisar las colecciones botánicas, y a aquellas personas que contribuyeron a la colección de esta información, como Álvaro Díaz, Eliseo Tesón, Juan Diego Ramírez, Helmar Cáceres Cárdenas; particularmente, a la directora de Asorquisan, Olga Marina Contreras, y a Alicia Rojas, por permitir realizar diferentes salidas en el departamento de Santander.

\section{Referencias}

[1] M. Chase, K. Cameron, J. Freudenstein, A. Pridgeon, G. Salazar, C. van den Berg, and A. Schuiteman, "An updated classification of Orchidaceae", Botanical Journal of the Linnean Society, vol. 177, pp. 151-174, 2015.

[2] J. Freudenstein, and W. Mark, "Phylogenetic relationships in Epidendroideae (Orchidaceae), one of the great flowering plant radiations: progressive specialization and diversification", $A n$ - 
nals of Botany, vol. 115, no. 4, pp. 665-681, 2015.

[3] J. Betancur, H. Sarmiento, L. Toro-González y J. Valencia, "Plan para el estudio y la conservación de orquídeas en Colombia", Universidad Nacional de Colombia, Facultad de Ciencias, Instituto de Ciencias Naturales y Ministerio de Ambiente, 2015.

[4] M. Bonilla, D. Yepes y J. Otero, "Diversidad y distribución de Catasetum rectangulare G.F. Carr (Orchidaceae: Catasetinae)", Orquideología, vol. 31, no. 1, pp. 15-25, 2014.

[5] M. Bonilla, C. Aguirre, D. Yepes, E. Gallego y J. Otero, "Catasetum (Orchidaceae: Catasetinae) en Colombia: lista actualizada", Revista de la Facultad de Ciencias Básicas, vol. 12, no. 1, pp. 22-35, 2016.

[6] M. Bonilla, J. Mosquera y J. Otero, "Biogeografía y Taxonomía de Catasetum", Memorias VII Congreso Colombiano de Botánica, pp. 252, 2013.

[7] G. Romero, "Subtribes Catasetinae", in Genera Orchidacearum, Vol. 5 Epidendroideae (Part two), A. Pridgeon, P. Cribb, M. Chase, and F. Rasmussen, Eds. New York: Oxford University Press, Oxford, 2009, pp. 11-12.

[8] G. Romero-González, "Las flores unisexuales y dimórficas de Catasetum Rich. (Orchidaceae)", Herbario CICY, vol. 4, pp. 32-36, 2012.

[9] M. Bonilla, J. Otero, E. Durán y C. Díaz, "Distribución espacial y clave taxonómica de Catasetinae (Orchidaceae) de Colombia”, Memorias VII Congreso Colombiano de Botánica, pp. 676, 2013.

[10] A. Holst, The world of Catasetums, Timber Press, Inc, 1999.

[11] S. Martínez, M. Bonilla y H. López "Listado de la flora Orchidaceae de Santander y comentarios sobre sus especies endémicas", Revista Facultad de Ciencias Básicas, vol. 11, n. ${ }^{\circ} 2$, pp. 54-111, 2015.

[12] P. Ortiz y G. Arango, "Catasetum lucis", Orquideología, vol. 19, n. ${ }^{\circ}$ 2, pp. 29-33, 1994.

[13] P. Ortiz, "Catasetum tricorne", Orquideología, vol. 20, n. ${ }^{\circ}$ 3, pp. 314-321, 1997.

[14] P. Ortiz y C. Uribe, Galería de orquídeas de Colombia (CD Edición), Bogotá: Asociación Bogotana de Orquideología, 2007.
[15] P. Ortiz y C. Uribe, Orquídeas, tesoro de Colombia $(A-D)$, Editorial Da Vinci Publicidad y Medios \& CIA. S. En. Colombia, pp. 397, 2014.

[16] R. Bernal, S. Grasdstein y M. Celis, Catálogo de plantas y líquenes de Colombia [online], Colombia, Instituto de Ciencias Naturales de Colombia, Bogotá, 2015. Disponible en: http://catalogoplantascolombia.unal.edu.co

[17] H. López, Orquídeas nativas de Santander. Bucaramanga: Gobernación de Santander, 2011.

[18] C. Jiménez, Orquídeas del Meta (CD Edición), Villavicencio, 2011.

[19] R. Hijmans, and D. Spooner, "Geography of wild potato species", American Journal Botany, vol. 88, pp. 2101-2112, 2001.

[20] R. Hijmans, S. Cameron, J. Parra, P. Jones, and A. Jarvis, "Very high resolution interpolated climate surfaces for global land areas", International Journal of climatology, vol. 25, pp. 1965-1978, 2005.

[21] IUCN, "Guidelines for application of IUCN Red List criteria at regional levels: Version 3.0", IUCN Species Survival Commission, IUCN, Gland, Switzerland and Cambridge. UK, 2003.

[22] E. Calderón-Sáenz, Libro Rojo de Plantas de Colombia. Volumen 3: "Orquídeas, Primera Parte", Serie Libros Rojos de Especies Amenazadas de Colombia. Bogotá: Instituto Alexander von Humboldt - Ministerio de Ambiente, Vivienda y Desarrollo Territorial, 2006.

[23] G. Dunsterville, Catasetum sanguineum Lindl. \& Paxton, American Orchid Society, 2010. Disponible en: http://www.aos.org/ Default.aspx?id=179

[24] G. Carnevali, I. Ramírez-Morillo, A. RomeroGonzález, C. Vargas, and E. Foldats, "Orchidaceae", in Flora of the Venezuelan Guayana, P. E. Berry et al. (eds.), vol. 7. Missouri Botanical Garden, St. Louis, 2003, pp. 200-619.

[25] F. Vallejo, M. Espitia, E. Estrada y H. Ramírez, Genética vegetal, Cali. Feriva-Universidad Nacional de Colombia, Sede Palmira, pp. 383, 2010.

[26] M. Bonilla, J. Otero y E. Durán, "Lista preliminar de Catasetinae (Orchidaceae) en Colombia". Memorias VII Congreso Colombiano de Botánica, pp. 5442013. 
[27] C. Murren, "Spatial and demographic population genetic structure in Catasetum viridiflavum across a human-disturbed habitat", Journal Evolution Biology, vol. 16, pp. 333-342, 2003.
[28] J. Sarmiento, "La familia Orchidaceae en Colombia", Actualidades biológicas, vol. 29 (Supl. 1), pp. 21-84, 2007. 